\title{
Antropometría y composición corporal en personas mayores de 60 años. Importancia de la actividad física
}

Heliodoro Alemán-Mateo, Lic. en N utr., ${ }^{(1)}$ Julián Esparza-Romero, M.C., ${ }^{(2)}$ Mauro E.Valencia, Ph.D. ${ }^{(3)}$

A lemán-Mateo H, Esparza-Romero J, Valencia ME. Antropometría y composición corporal en personas mayores de 60 años. Importancia de la actividad física. Salud Publica Mex 1999;41:309-316.

\section{Resumen}

Objetivo. Medir los indicadores antropométricos de composición corporal por bioimpedancia eléctrica y la actividad física, así co mo la glucosa sanguínea y la presión arterial en personas mayores de 60 años de una región rural. Ma terial y métodos. Por muestreo intencional no probabilístico se seleccionaron 54 sujetos mayores de 60 años, quienes se sometieron al protocolo estandarizado para las mediciones antropométricas y composición corporal por bioimpedancia eléctrica (BIE) y actividad física mediante el cuestionario de actividades. Asimismo, se midió la presión arterial y se realizó la prueba de tolerancia a la glucosa. Los valores promedio se analizaron por sexo mediante la prueba t de Student, y la asociación de la actividad física y la composición corporal con otras variables, por regresión lineal simple y el coeficiente de correlación de Pearson. $\mathbf{R e}$ sultados. La actividad física correlacionó negativamente con el porcentaje de grasa $(r=-0.36 ; p=0.007)$; la presión arterial diastólica $(r=-0.36 ; p=0.006)$ y sistólica $(r=-0.32 ; p=$ 0.018 ) y el índice de masa corporal, con la glucosa posdosis $(r=0.31 ; p=0.024)$. Conclusiones. La actividad física en este grupo de personas puede tener un impacto favorable respecto a los indicadores de salud tan importantes que se mencionaron.

Palabras clave: anciano; antro pometría; composición corporal; aptitud física; México

\author{
A lemán-Mateo H, Esparza-Romero J, Valencia ME. \\ Anthropometry and body composition \\ in elderly people. \\ Importance of physical activity. \\ Salud Publica Mex 1999;41:309-316.
}

\begin{abstract}
A bstract
Objective.To measure anthropo metric indicators of body composition by electric bioimpedence, as well as physical activity, blood glucose and arterial pressure in rural people over 60 years of age. Material and methods.A total of 54 subjects over 60 years old were selected by intentional nonprobabilistic sampling. They were subjected to a standardized protocol to measure anthropometric indicators, body composition by electric bioimpedance (EBI), and physical activity was deter mined by an activity questionnaire.A rterial pressure and glucose tolerance levels were also measured. A nalysis included Student's t test to detect differences by sex and the association of physical activity, body composition and other variables by simple linear regression and Pearson's correlation coefficient. Results Physical activity correlated negatively with percent fat $(r=-0.36 ; p=0.007)$ and diastolic $(r=-0.36 ; p=0.006)$ and systolic blood pressure $(r=-0.32 ; p=0.018)$, and body mass index with post dose glucose $(r=0.31 ; p=0.024)$. Conclusions Physical activity in this age group may have a favorable impact with respect to the mentioned health indicators.
\end{abstract}

Key words: aged; anthropometry; body composition; physical fitness; Mexico

(1) Estudiante de Doctorado. División de N utrición, Centro de Investigación y Desarrollo A.C. (CIAD, A.C.)

(2) Profesor-Investigador Asociado B. División de N utrición, Centro de Investigación y Desarrollo A.C. (CIAD, A.C.)

(3) Director de la División de Nutrición. División de Nutrición, Centro de Investigación y Desarrollo A.C. (CIAD, A.C.)

Fecha de recibido: 9 de septiembre de 1998 - Fecha de aprobado: 11 de febrero de 1999

Solicitud de sobretiros: Dr. Mauro E.Valencia. Centro de Investigación en Alimentación y D esarrollo, CIAD,A.C.

Carretera a laVictoria km. 0.6,A partado Postal 1735, 83000 Hermosillo, Sonora, México.

Correo electrónico: mauro@ cascabel.ciad.mx 
$\mathrm{E}$ n la actualidad, tanto en los países industrializados como en los que están en vías de desarrollo, se ha registrado un incremento importante de la población mayor de 60 años. De acuerdo con el Instituto Nacional de Estadística, Geografía e Informática, en 1990 en México la población de la tercera edad representó $6.1 \%$ de la población total. ${ }^{1}$ Se estima que esa cifra aumentará a $7.7 \%$ para el año $2010 .{ }^{2}$ En las personas de la tercera edad se presentan diversos cambios biológicos y su estilo de vida también se modifica. Indudablemente, los cambios antropométricos y de composición corporal relacionados con la edad cobran más interés cada día por su relevancia e implicaciones en el estado de nutrición, en la respuesta al apoyo nutricio y farmacológico, en la capacidad funcional, en el pronóstico y tratamiento de pacientes hospitalizados, así como respecto a factores de riesgo para el desarrollo de enfermedades crónico-degenerativas.

Hoy en día, en el adulto mayor se reconoce la importancia de los cambios en peso, en talla y en composición corporal. En algunos estudios ${ }^{3,4}$ se ha informado que el peso corporal aumenta entre los 20 y los 50 años de edad, y que después de los 70 años disminuye progresivamente. Respecto a la talla, se ha indicado que ésta disminuye en la medida en que aumenta la edad. ${ }^{5-7}$ Asimismo se ha establecido que, en las personas de la tercera edad se presentan otros cambios antropométricos como el aumento en el grosor de los pliegues cutáneos, la relación cintura-cadera y el índice de masa corporal (IMC), entre otros. ${ }^{8,9}$ Respecto a los cambios en la composición corporal se ha mostrado que la masa corporal libre de grasa (MCLG) disminuye de 25 a 30\% entre los 30 y los 70 años de edad, y ocurre un aumento de la grasa corporal de 10 a 15\% durante todo el ciclo de vida. ${ }^{10-12}$ En algunos estudios se ha encontrado que, además de aumentar, la grasa corporal se redistribuye de manera desfavorable para la salud del adulto mayor; aumenta la cantidad de tejido adiposo en la parte central del cuerpo, ${ }^{13,14}$ lo cual puede ser un importante factor de riesgo para el desarrollo de enfermedades crónicas y alteraciones metabólicas como hipercolesterolemia, resistencia a la insulina, aterosclerosis, hipertensión y diabetes mellitus. ${ }^{15}$

Por otro lado, se ha reportado que la disminución de la MCLG y el aumento de la grasa corporal en este grupo de edad se deben a las alteraciones hormonales y de los mediadores hormonales, así como a la reducción de la actividad física. ${ }^{16}$ En el anciano también se acentúan otros cambios como la disminución de la tasa metabólica basal, las alteraciones en el metabolismo de la glucosa y de los lípidos. ${ }^{17}$ Todos estos cambios hacen al adulto mayor más vulnerable al desarrollo, o complicaciones de diversas enfermedades, que comprometen su estado de salud y la capacidad funcional. En México existen pocos estudios referidos sobre antropometría, composición corporal y actividad física, y sobre todo acerca de la posible relación entre la actividad física con la presión arterial y la composición corporal, y de esta última con la glucosa sanguínea. El objetivo de este estudio fue medir algunos indicadores antropométricos, de composición corporal por bioimpedencia eléctrica (BIE) y actividad física, así como la glucosa sanguínea y la presión arterial en personas mayores de 60 años y con vida libre irrestricta en una comunidad rural del noroeste de México.

\section{Material y métodos}

Por muestreo intencional no probabilístico se seleccionaron 54 sujetos mayores de 60 años, con una gran diversidad de actividades físicas, orientados en espacio y tiempo y con deseos de participar; todos pertenecen al ejido La Victoria, comunidad rural situada a $7 \mathrm{~km}$ de la ciudad de Hermosillo, Sonora, México.

A cada individuo se le explicó detenidamente el protocolo de estudio, y después se le invitó a participar. Durante la primera entrevista se les preguntó acerca de su estado de salud para descartar a los sujetos con enfermedades como cáncer, infarto al miocardio, angina de pecho, artritis reumatoide y diabetes tipo 2 con tratamiento de insulina. Ningún sujeto tomó medicamentos (que se reconoce influyen en el agua corporal total) durante el estudio; todos refirieron haber tenido un peso estable durante los seis meses previos y ninguno presentó edema o retención de líquidos. La tarde previa a las mediciones, los sujetos residieron en la unidad metabólica de la división de nutrición humana, donde se les brindó una cena con un platillo típico regional, el cual cubrió una tercera parte de su requerimiento de energía y contenía una distribución porcentual de la energía de 15\% de proteínas, 30\% de grasas y $55 \%$ de carbohidratos. También se vigiló que no tomaran agua, café o té, en las horas previas a las mediciones. A la mañana siguiente los sujetos se sometieron al protocolo para las mediciones antropométricas, de composición corporal y de actividad física, así como para la prueba de tolerancia a la glucosa y la toma de presión arterial.

El peso se registró con una balanza electrónica digital de 0 a 150x0.05 kg de capacidad (ADN FV-150 K, Japón), y la talla, con un estadiómetro Holtain (Holtain Limited, Dyfed. Britain). Los panículos adiposos (bicipital, tricipital, subescapular y suprailíaco) se midieron con un plicómetro (Holtain LTD. Crymych 
U.K.). Todas las variables antropométricas se midieron de acuerdo con la técnica de Durnin..$^{18}$ La altura talón-rodilla se midió con un estadiómetro portátil (Holtain LTD, U.K.) según la técnica de Chumlea y colaboradores. ${ }^{19}$ Se calculó asimismo el IMC y se diagnosticó el estado energético de acuerdo con los nuevos criterios de la Organización Mundial de la Salud (OMS). ${ }^{20}$ La deficiencia crónica de energía se diagnosticó en correspondencia con los criterios de James y colaboradores, ${ }^{21}$ los cuales consideran el IMC y el nivel de actividad física (NAF).

La composición corporal se evaluó por bioimpedancia eléctrica con el sistema RJL (RJL Systems Detroit, Mich., Estados Unidos de América, EUA). Las mediciones se realizaron de acuerdo con la técnica de Lukaski y colaboradores. ${ }^{22}$ La MCLG y la grasa corporal se determinaron con la ecuación específica para la población mayor de 60 años, propuesta por Deuerenberg y colaboradores, ${ }^{23}$ que fue validada en mujeres ${ }^{24}$ y en hombres. ${ }^{25}$

La prueba de tolerancia a la glucosa se llevó a cabo en la unidad metabólica del CIAD bajo condiciones controladas y de acuerdo con la técnica reportada por Schulz y Weidensee. ${ }^{26} \mathrm{El}$ diagnóstico de intolerancia a la glucosa y diabetes tipo 2 se realizó según la clasificación de la $\mathrm{OMS}^{27}$ considerando los valores en sangre capilar, y se verificó con los criterios del Comité de Expertos sobre el Diagnóstico y Clasificación de la Diabetes Mellitus. ${ }^{28}$

La presión arterial se midió en condiciones de reposo con un esfigmomanómetro de columna de mercurio (Desk Model Adult Calibrated 0320, distribuido por Graham-Field Inc., Nueva York, EUA, y fabricado en Japón). Se midió la presión arterial y se llevó a cabo el diagnóstico de hipertensión arterial siguiendo las recomendaciones y criterios de la OMS. ${ }^{29}$

La actividad física se evaluó mediante la metodología del cuestionario que desarrollaron y validaron Kriska y colaboradores ${ }^{30}$ y utilizada en personas de la tercera edad. ${ }^{31}$ El gasto energético expresado en NAF se calculó con los múltiplos de metabolismo basal o NAF de las diversas actividades reportadas en la población adulta ${ }^{32,33} \mathrm{y}$ de la tercera edad. ${ }^{34,35}$ El comité de expertos en proteínas y energía de la FAO/OMS/UNU de 1985 sugirió esta forma de expresión de la actividad física, y se utiliza generalmente para expresar este componente del gasto energético en estudios de fisiología y epidemiología de la nutrición. Cabe aclarar que el NAF es el resultado de dividir el gasto diario de energía por actividad física en kcal o kJ/ día, entre el gasto por metabolismo basal expresado en las mismas unidades. ${ }^{36}$ En el cuestionario se incluyeron las actividades laborales y discrecionales realizadas durante los 12 meses previos a la aplicación. A cada sujeto se le preguntó acerca de cada actividad que llevaba a cabo y el tiempo que empleaba en desarrollarla. Se calculó el tiempo expresado en horas por cada una de las actividades registradas, así como el NAF. Para fines del presente trabajo, el NAF representa el gasto energético por actividad física de un día promedio, representativo del año.

Los datos se analizaron en el paquete estadístico NCSS 97 (Statistical System for Windows). Los resultados de los análisis de cada una de las variables se reportan como media y desviación estándar. Las diferencias de medias por sexo se analizaron por la prueba $t$ de Student para dos muestras independientes.

La asociación entre la actividad física y la composición corporal con el resto de las variables se analizó por regresión lineal simple y se obtuvo el coeficiente de correlación de Pearson. En ambas pruebas se consideró el nivel de significancia de $p<0.05$.

Todos los procedimientos descritos en este estudio fueron aprobados por el Comité Interno-Externo de Ética del Centro de Investigación en Alimentación y Desarrollo, A.C.

\section{Resultados}

\section{Características físicas y antropométricas}

Se estudiaron 54 sujetos mayores de 60 años de edad, todos residentes de una región rural del estado de Sonora, México; participaron 22 mujeres y 32 hombres con una edad promedio de 68 años. Las variables se analizaron por sexo, mediante una prueba $t$ de dos muestras independientes. En los hombres los valores promedio de talla y altura talón-rodilla fueron mayores comparados con los de las mujeres $(p<0.001)$. Contrariamente, en las mujeres los cuatro pliegues cutáneos fueron mayores $(p<0.001)$, con excepción del pliegue cutáneo subescapular, el cual no mostró diferencias. En el resto de las variables antropométricas analizadas no se encontraron diferencias significativas por sexo. Los resultados se presentan en el cuadro I.

Por otra parte, con el IMC se obtuvo el estado de nutrición: 38.8\% de los sujetos (11 mujeres y 10 hombres) clasificaron como preobesos, y 18.5\% (cinco mujeres y cinco hombres), con obesidad grados I o II. El resto de la muestra se clasificó en la categoría normal $(38.8 \%)$ y bajo peso (3.7\%). Un sujeto presentó un IMC de 16 y un NAF superior a 1.4. De acuerdo con los criterios de James y colaboradores, ${ }^{21}$ este sujeto se diagnosticó con deficiencia crónica de energía grado I. Los resultados se presentan en el cuadro II. 


\section{Cuadro I \\ EdAD Y dATOS ANTROPOMÉTRICOS DE PERSONAS MAYORES DE 60 AÑOS DE UNA REGIÓN RURAL DEL NOROESTE DE MÉXICO, 1997}

\begin{tabular}{lccc} 
& Mujeres $(\mathrm{n}=22)$ & Hombres $(\mathrm{n}=32)$ & $\mathrm{p})$ \\
Edad (años) & $68.8(65.5-72.1)$ & $68.6(66.2-70.9)$ & 0.92 \\
\hline Peso $(\mathrm{kg})$ & $66.3(61.1-71.5)$ & $71.9(66.4-77.4)$ & 0.15 \\
\hline Talla $(\mathrm{m})$ & $1.55(1.53-1.58)$ & $1.67(1.66-1.69)$ & $0.001^{*}$ \\
\hline IMC (peso kg/talla $\left.{ }^{2}\right)$ & $27.2(25.4-29.0)$ & $25.5(23.8-27.3)$ & 0.18 \\
\hline Talón-Rodilla $(\mathrm{cm})$ & $48.2(46.9-49.5)$ & $52.5(51.8-53.2)$ & $0.001^{*}$ \\
\hline Pliegue cutáneo bicipital $(\mathrm{mm})$ & $14.1(11.3-16.9)$ & $5.1(4.1-6.2)$ & $0.001^{*}$ \\
\hline Pliegue cutáneo tricipital $(\mathrm{mm})$ & $21.6(18.1-25.2)$ & $11.1(9.4-12.8)$ & $0.001^{*}$ \\
\hline Pliegue cutáneo subescapular $(\mathrm{mm})$ & $17.8(14.9-20.7)$ & $15.4(13.2-17.6)$ & 0.17 \\
\hline Pliegue cutáneo suprailíaco $(\mathrm{mm})$ & $21.7(18.8-24.5)$ & $8.4(7.3-9.4)$ & $0.001^{*}$ \\
\hline Circunferencia de cadera $(\mathrm{cm})$ & $101.6(96.1-105.2)$ & $96.2(93.6-98.8)$ & 0.68 \\
\hline Circunferencia de cintura $(\mathrm{cm})$ & $98.3(92.5-104.0)$ & $95.1(89.1-101-2)$ & 0.47 \\
\hline Relación cintura-cadera & $0.97(1.00-1.02)$ & $0.98(0.94-1.03)$ & 0.70
\end{tabular}

IMC: índice de masa corporal

* Diferencias significativas por sexo $p<0.001$

$\mathrm{N}$ ota: los valores promedio se presentan como medias (intervalos de confianza al $95 \%$ )

\section{Composición corporal}

En este estudio las mujeres presentaron un mayor porcentaje de grasa corporal y menor MCLG que los hombres $(p<0.001)$. De acuerdo con los resultados del análisis de regresión lineal simple y la prueba de correlación de Pearson, el porcentaje de grasa en las mujeres correlacionó significativamente con la circunferencia de la cintura $(r=0.74 ; p<0.001)$, peso corporal $(r=0.71 ; p<0.001)$, IMC $(r=0.69 ; p<0.001)$, pliegue cutáneo suprailíaco $(r=0.66 ; p<0.001)$, tricipital $(r=0.61$; $p<0.001)$ y subescapular $(r=0.52 ; p<0.001)$, y la edad $(r=0.54 ; p<0.001)$. En los hombres el porcentaje de grasa también correlacionó con el peso corporal $(r=0.84$; $p<0.001)$, el IMC $(r=0.80 ; p<0.001)$, el pliegue cutáneo tricipital, $(r=0.69 ; p<0.001)$, la presión arterial diastólica $(r=0.66 ; p<0.001)$, el pliegue cutáneo subescapular $(r=0.66 ; p<0.001)$, la cintura $(r=0.66 ; p<0.001)$, el pliegue cutáneo suprailiaco $(r=0.60 ; p<0.001)$, la presión arterial diastólica $(r=0.56 ; p<0.001)$, y con la relación cintura-cadera $(r=0.47 ; p<0.001)$. Los resultados de la composición corporal por BIE y otras variables analizadas se presentan en el cuadro III.

\section{Tolerancia a la glucosa}

Con los resultados de la prueba de la tolerancia a la glucosa nueve mujeres y 21 hombres $(55.5 \%)$ se clasificaron como normales. Otras seis mujeres y cinco hom-

\section{Cuadro II}

ESTADO DE SALUD DE LAS PERSONAS MAYORES DE 60 AÑOS, RESIDENTES DE UNA REGIÓN RURAL DEL NOROESTe de MéXıCO, 1997

$$
\text { Mujeres Hombres Ambos }
$$

Tolerancia a la glucosa

\begin{tabular}{lrrl} 
Sujetos normales & 9 & 21 & 30 \\
\hline Sujetos con intolerancia & 6 & 5 & 11 \\
\hline Sujetos con diabetes & 7 & 6 & 13 \\
\hline Total & & & 54
\end{tabular}

Presión arterial

\begin{tabular}{lrrr} 
Normotensos & 13 & 25 & 38 \\
\hline Hipertensos & 9 & 7 & 16 \\
\hline Total & & & 54
\end{tabular}

Estado de nutrición por IMC

\begin{tabular}{lrrr} 
Bajo peso & 1 & 1 & 2 \\
\hline N ormal & 5 & 16 & 21 \\
\hline Preobeso & 11 & 10 & 21 \\
\hline O besidad grado I & 4 & 3 & 7 \\
\hline O besidad grado II & 1 & 2 & 3 \\
\hline O besidad grado III & 0 & 0 & 0 \\
\hline Total & & & 54
\end{tabular}

* Frecuencia del número de personas por sexo en cada categoría de la prueba 


\section{Cuadro III}

\section{Composición corporal, gluCosa sanguínea, Nivel de ACtividAd física en PERSONAS MAYORES de 60 años, de una región RURAL del nOROeste de México, 1997}

\begin{tabular}{|c|c|c|c|}
\hline & M ujeres $(n=22)$ & Hombres $(n=32)$ & $p$ \\
\hline \multicolumn{4}{|l|}{ Composición corporal por BIE } \\
\hline MCLG (kg) & $32.1(30.2-34.1)$ & $43.3(41.5-45.1)$ & $0.001^{\ddagger}$ \\
\hline Grasa corporal (\%) & $50.7(48.0-53.5)$ & $38.4(35.8-41.1)$ & $0.001^{\ddagger}$ \\
\hline Grasa corporal (kg) & $34.1(30.2-38.1)$ & $28.5(24.6-32.6)$ & $0.05^{5}$ \\
\hline \multicolumn{4}{|c|}{ Glucosa en sangre capilar (mg/dl)* } \\
\hline Ayuno & $100(86-117)$ & $98(87-110)$ & 0.81 \\
\hline Posdosis & $164(135-201)$ & $138(118-162)$ & 0.16 \\
\hline \multicolumn{4}{|l|}{ Presión arterial $(\mathrm{mmHg})$} \\
\hline Sistólica & $147(140-153)$ & 137 (129-144) & $0.04^{5}$ \\
\hline Diastólica & $75(72-79)$ & $77(73-81)$ & 0.52 \\
\hline \multicolumn{4}{|l|}{ Actividad física } \\
\hline $\mathrm{N}$ ivel de actividad física & $1.50(1.45-156)$ & $1.68(1.54-1.81)$ & $0.036^{5}$ \\
\hline $\begin{array}{l}\text { BIE: bioimpedencia eléctrica } \\
\text { MCLG: masa corporal libre d } \\
\text { * Media geométrica (intervalo } \\
\text { q D iferencias significativas por } \\
\text { \& D iferencias significativas por } \\
\text { N ota: los valores promedios }\end{array}$ & ntervalos de confian & & \\
\hline
\end{tabular}

bres $(20.4 \%)$ presentaron intolerancia a la glucosa. De la muestra, siete mujeres y seis hombres $(24.1 \%)$ presentaron diabetes mellitus no insulino dependiente o tipo 2 (cuadro II). Al analizar por sexo los valores de glucosa en ayuno y posdosis, no se encontraron diferencias significativas (cuadro III).

\section{Presión arterial}

De acuerdo con las cifras registradas de presión arterial y la clasificación de la OMS, $29 \%$ de los sujetos (nueve mujeres y siete hombres) presentaron algún grado de hipertensión arterial; el resto de la muestra se clasificó como normotenso (cuadro II). Al analizar los valores de presión arterial por sexo se encontraron diferencias significativas; sin embargo, la presión arterial sistólica fue menor en los hombres $(p<0.05)$ (cuadro III).

\section{Actividad física}

En las mujeres, el NAF fue de 1.50, y representa la energía que gastaron en diversas actividades como lavar a mano, planchar, hacer tortillas, cortar y cargar leña, barrer, y preparar comida, entre otras. En los hombres la media del NAF fue de 1.65. Al observar el patrón de actividad física los hombres realizaron tareas que de- mandaron un mayor gasto energético como la albañilería, la cosecha de frutas y hortalizas, cortar leña y reparación de cercos, entre otras. Cuando los valores promedio del NAF se compararon por sexo, se observó que en los hombres fue mayor que en las mujeres $(p<0.05)$ (cuadro III).

Finalmente, al analizar la actividad física en los 54 sujetos se observó una correlación inversa entre el NAF y el porcentaje de grasa corporal total $(r=-0.36$; $p=0.007)$ (figura 1), la presión arterial diastólica $(r=$ $-0.36 ; p=0.006)$ y sistólica $(r=-0.32 ; p=0.0018)$, así como con el pliegue cutáneo bicipital $(r=-0.27 ; p=0.044)$ y el $\operatorname{IMC}(r=-0.26 ; p=0.05)$.

\section{Discusión}

Una limitante de este trabajo es que el número de participantes impide profundizar en ciertos análisis, debido a que esta indagación es la primera parte de un estudio más amplio.

Los valores promedio del peso y la talla, así como de los demás indicadores antropométricos medidos, son casi similares a los valores que se han indicado en otro estudio con población urbana del centro del país. ${ }^{37}$

De la población estudiada, 38.8\% clasificó como preobesa (IMC $=25-29.9)$, y 18.5\%, como obesa (IMC $>30$ ), según la clasificación de la $\mathrm{OMS}^{20}$ y respecto a la 


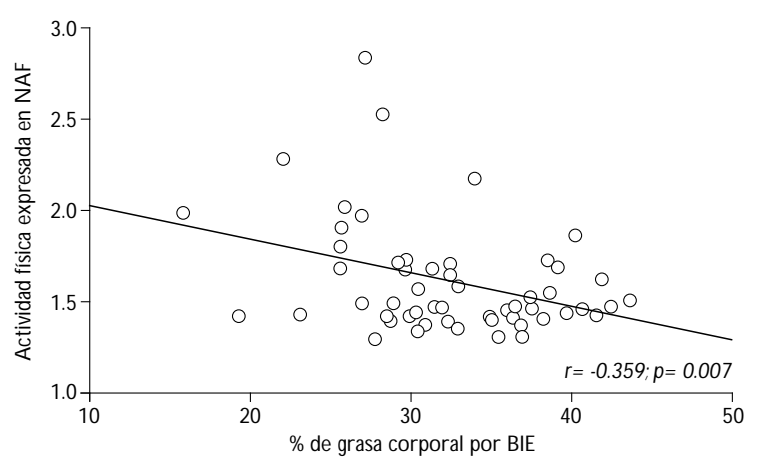

Figura 1. Correlación entre la actividad física y la GRASA CORPORAL EN PERSONAS MAYORES DE 60 AÑOS DE una región rural del nOROeste de México, 1997

cual es conveniente hacer algunas observaciones: Mattila y colaboradores ${ }^{38}$ encontraron que los hombres de 80 años tuvieron una sobrevida de cinco años cuando el IMC fue de 30, así que en el anciano encontrar obesidad grado I o II puede ser un factor protector y no de riesgo como lo indica la OMS. ${ }^{20}$ Sin embargo, sería incorrecto extrapolar los resultados de Mattila y colaboradores ${ }^{38}$ a este estudio, debido a que sólo se incluyeron cinco personas mayores de 80 años. Por otra parte, Andres y colaboradores ${ }^{39}$ encontraron que en los hombres de 60 a 69 años la mortalidad más baja se observó cuando había un IMC de 26.6, mientras que en las mujeres la mortalidad más baja se presentó con un IMC de 27.3. Al tomar en cuenta que son insuficientes los datos sobre los puntos de corte más convenientes respecto a la sobrevida y la mortalidad para la población mayor de 60 años, se recurrió a los criterios del Committee on Diet and Health, del Food Nutrition Board ${ }^{40}$ que indican el punto de corte de 25-29 como deseable, y de acuerdo con el cual los sujetos clasificados como obesos se consideraron normales.

Los resultados de la composición corporal por BIE mostraron que las mujeres tuvieron una mayor cantidad de grasa que los hombres, lo cual concuerda con los resultados de otros estudios. ${ }^{9,41} \mathrm{El}$ porcentaje de grasa reportado por Broekhoff y colaboradores ${ }^{24}$ en una población femenina holandesa, fue menor al que se encontró en las mujeres de este estudio (40\% vs. 50.7\%).

Cuando se analizó el porcentaje de grasa respecto de la presión arterial, no se observó una correlación significativa; sin embargo, al analizar el IMC como un indicador de tamaño corporal con la presión arterial sistólica en los 52 sujetos, sí se encontró una asocia- ción significativa $(r=0.40 ; p=0.003$ y $r=0.46 ; p=0.0004$, respectivamente); esto es consistente con lo que se indica en otros estudios. ${ }^{42,43}$ En general, los resultados sugieren que las tasas elevadas de hipertensión en la tercera edad pueden modificarse al aumentar la actividad física y disminuir el peso corporal.

Por otro lado, en algunos estudios se ha encontrado que las personas activas tienen menor riesgo de padecer diabetes mellitus tipo $2 .^{44,45}$ En este estudio no se encontró relación alguna entre los niveles de glucosa (ayuno y posdosis) con la actividad física y la grasa corporal. Sin embargo, sí se observó una correlación de la glucosa posdosis con el IMC $(r=0.31 ; p=0.024)$ y con la circunferencia de la cintura $(r=0.36 ; p=0.008)$. La relación positiva entre el IMC y la glucosa posdosis es consistente con los resultados que han informado King y colaboradores. ${ }^{46}$ En relación con la circunferencia de la cintura, evidencias recientes sugieren que dicha medición puede proporcionar una correlación más práctica de la distribución de la grasa abdominal y la morbilidad y mortalidad en general. La circunferencia de la cintura es un índice aproximado de la masa grasa intrabdominal y de la grasa corporal total. Por otra parte, la asociación entre la circunferencia de cintura y la glucosa podría ser un factor importante en la intolerancia a la glucosa que se detectó en los sujetos estudiados. Se ha reportado que la grasa intrabdominal y la obesidad per se se asocian con intolerancia a la glucosa y con resistencia a la insulina, y ambas alteraciones son factores de riesgo para el desarrollo de la diabetes mellitus tipo $2 .^{20}$

Con la prueba de tolerancia a la glucosa se diagnosticó diabetes tipo 2 a $24 \%$ de los sujetos de la muestra, lo cual es similar a la prevalencia naciona ${ }^{47}$ y a la que informan Castro y colaboradores ${ }^{48}$ para la población mayor de 60 años. Otro 20\% de la muestra presentó intolerancia a la glucosa. De acuerdo con el Comité de Expertos sobre el Diagnóstico y Clasificación de la Diabetes Mellitus, ${ }^{28}$ en general los sujetos diagnosticados con intolerancia a la glucosa progresan a la diabetes en proporción de 5\% al año. Estos resultados señalan una tasa elevada de sujetos ancianos prediabéticos, lo cual sugiere un aumento en la incidencia de diabetes en la población de la tercera edad a largo plazo.

En este trabajo se estimó el NAF utilizando la metodología del cuestionario de actividades que desarrollaron y validaron Kriska y colaboradores. ${ }^{30}$ En las mujeres, el NAF fue de 1.50 y representa la energía que gastaron en diversas actividades como lavar a mano, planchar, hacer tortillas, cortar y cargar leña, barrer y preparar comida, entre otras. En los hombres la media del NAF fue de 1.65. Al observar el patrón de actividad física, los hombres realizaron actividades que 
demandaron un mayor gasto energético como albañilería, cosecha de frutas y hortalizas, cortar leña y construir cercas, entre otras. Ni los hombres ni las mujeres indicaron realizar actividades deportivas. Cuando los valores promedio del NAF se compararon por sexo, se observó que el nivel de actividad física fue mayor en los hombres respecto al de las mujeres $(p<0.05)$.

Por otro lado, se ha reportado que la actividad física en edades mayores se asocia con una tasa más baja en la mortalidad general y por enfermedades cardiovasculares. ${ }^{49-51}$ En este estudio, al analizar la actividad física expresada en NAF con la composición corporal por BIE en los 54 sujetos, se encontró una correlación inversa entre el NAF y el porcentaje de grasa, así como con la presión arterial sistólica y la diastólica. Este último resultado es consistente con el que han informado Teo e Idris. ${ }^{42}$ En relación con la actividad física en las mujeres, se observó una correlación positiva entre el NAF y la MCLG $(r=0.54 ; p=0.009)$, así como con el peso corporal $(r=0.61 ; p=0.002)$.

\section{Conclusiones}

Los resultados de los análisis muestran que la actividad física en las personas de la tercera edad puede tener un impacto favorable sobre la composición corporal, en especial sobre la grasa corporal y la presión arterial (sistólica y diastólica) en el grupo estudiado. Sin embargo, a pesar de que los resultados parecen destacar la importancia que representa la actividad física en relación con la composición corporal de este grupo en particular, una limitación natural por el diseño de corte transversal impide inferir que la cantidad de grasa se relacione con el nivel de actividad física o que los sujetos con menos grasa sean más activos. Por otro lado, la mayoría de los sujetos estudiados se ubican entre normales y preobesos, lo cual pudiera relacionarse con el hecho de que provienen de una zona rural, con un patrón de actividad física relativamente alto.

\section{A gradecimientos}

Expresamos nuestro más sincero agradecimiento a todos los voluntarios que participaron incondicionalmente en este estudio. Agradecemos la valiosa participación por su apoyo técnico a la licenciada en Nutrición Thelma Reza Durán, la químico-bióloga Bertha I. Pacheco, la químico-bióloga Alma Robles, el químicobiólogo Fernando Leyva Livshin y a la médica cirujano María Eugenia Cardiel, así como al resto del personal del CIAD que colaboró de alguna manera en la realización de este trabajo.

\section{Referencias}

1. Instituto Nacional de Estadística, Geografía e Informática. La tercera edad en México, 1993. IX Censo General de Población yVivienda. México, D.F: IN EGI, 1990.

2. Secretaría de Salud. La salud de la población de edad avanzada. Un reto más hacia el futuro. Cuadernos de salud núm. 1 Población y Salud. México, D.F.SSA, 1994.

3. Borkan GA, Hults DE, Gerzof SG, Robbins AH, Silbert CK.A ge changes in body composition revealed by computed tomography. J Gerontol 1983;38:673-677.

4. Silver AJ, G uillen CP, Kahl MJ, M orley JE. Effect of aging on body fat. J Am Geriatr Soc 1993;41:211-213.

5. N ajjar MF, Rowland M. N ational C enter for Health Statistics. Anthropometric reference data and prevalence of overweight, United States, 19761980. W ashington, D.C: US G overnment Printing 0 ffice, (Vital and health statiscs series 11 DHHS publication [PHS] 87-1699)1987 .

6.Stoudt HW, D amon A, M CFarlan R, Roberts J.W eight, height, and selected body dimensions of adults. United States, 1960-62. Washington, DC: US $\mathrm{N}$ ational Center for Health Statistics, Government Printing 0 ffice, 1965. (Vital and health statiscs series 11 DHEW publication [HRA] 76-1074). 7. N ational Center for Health Statistics, N ajjar MF, Kuczmarski RJ.Anthropometric reference data and prevalence of overweight, $\mathrm{H}$ ispanic $\mathrm{H}$ ealth and N utrition Examination Survey, 1982-84.W ashington, D.C.: US G overnment Printing 0 ffice 1989 (Vital and health statistics series 11).

8. Abraham S, Johnson CL, N ajjar MF.W eight and height of adults 18-74 years of age. Rockville MD: N ational Center for Health Statistics (Vital and health statiscs series 11: \#211 DHEW publication [PHS] 79-1659). 9. Durnin JVGA, Womersley J. Body fat assessed from total body density and its estimation from skinfold thickness: Measurements on 481 men and women aged from 16-72 years. Br J N utr 1974;32:77-96.

10. Fleg J, Lakatta. Role of muscle loss in the age-associated reduction in V02 max. J Appl Physiol 1988;65:1147-1151.

11. Flynn MA, Nolph GB, Baker AS, Martin W M, Krause G. Total body potassium in aging humans: A longitudinal study. Am J Clin Nutr 1989; 50:713-717

12. Grimby G, Saltin B. Mini review: The aging muscle. Clin Physiol 1983;3:209-218.

13. Scwartz RS, Shuman W P, Bradbury VL, Clain KC, Fellinghan GW, Beard $\mathrm{JC}$ et al. Body fat distribution in healthy young and older men.J Gerontol 1990;45:M 181-185.

14. Kohrt W M, Malley MT, Dalsky GP, Holloszy JO. Body composition of healthy sedentary and trained, young and older men and women. Med Sci Sports Exerc 1992;24:832-837.

15. D espres JP, Moorjani S, Lupien PJ, Tremblay A, N adeau A, Bouchard C. Regional distribution of body fat, plasma lipoproteins and cardiovascular disease. Asteriosclerosis 1990;10:487-511.

16. Roubenoff R, Rall LC. Humoral mediation of changing body composition during aging and chronic inflammation. N utr Rev 1993;51:1-11.

17. Shock N W. The physiology of aging. Sci Am 1962;206:100-108.

18. Durnin JVGA. Anthropometric methods for assessing nutritional status. En: Horwitz, Mac Fadyen, Munro, Srcrimshaw, Steen, W illiams eds. N utrition in the elderly. 0 xford: 0 xford University Press, 1989:15-32.

19. ChumleaW C, Roche AF, Steinbaugh ML. Estimating stature from knee height for persons 60 to 90 years of age. J Am Geriatr Soc 1985;33:116120.

20. Report of aW HO consultation on obesity. Preventing and managinG the global epidemic. Ginebra:W orld Health O rganization, 1997.

21. James W PT, Ferro-Luzzi A,W aterlow JC. D efinition of chronic energy deficiency in adults. Eur J C lin N utr 1988:42:969-981. 
22. Lukasky HC, Johnson PE, Bolonchuck W W, Lykken GI. Assessment of fat free mass using bio-electrical impedance measurements of human body. Am J C lin N utr 1985;41:810-817.

23. Deuerenberg P,Van der Kooy K, Leenen R, Evers P, Hulshof T. Assessment of body composition by bioelectrical impedance in a population aged $>60$ years. Am J Clin N utr 1990;51:3-6.

24. Broekhoff $C$, Voorrips LE, W eijenberg MP, W itvoet GA, van Staveren $W A$, D euerenberg P. Relative validity of different methods to assess body composition in apparently healthy elderly women. Ann Nutr Metab 1992;36:148-156.

25. Fuller NJ, Sawyer MB, Laskey MA, Paxton P, Elia M. Prediction of body composition in elderly men over 75 years of age. Ann Human Biol 1996;23:127-147.

26. Schulz LO,W eidensee RC. G lucose tolerance and physical activity in a Mexican indigenous population. Diabetes Care 1995;18:1274-1276.

27.W orld Health 0 rganization. Diabetes Mellitus. Report of aW HO Study Group. Ginebra:W HO (Technical Report Series núm. 727), 1985.

28. The expert Committee on the Diagnosis and Classification of Diabetes Mellitus. Report expert committee on the diagnosis and classification of diabetes mellitus. Diabetes Care 1997;20:1183-1197.

29. World Health O rganization. Arterial hypertensión. Ginebra: Expert Committee Report, (Technical Report núm. 628), 1978.

30. Kriska AM, Knowler W C, LaPorte RE, D rash AL,W ing RR, Blair SN et al. Development of questionnaire to examine relationship of physical activity and diabetes in Pima Indians. Diabetes $C$ are 1990;13:401-411.

31. Alemán-Mateo H, Esparza-Romero J,Valencia-Juillerat ME. Requerimiento de energía en personas activas de la tercera edad, residentes de una región rural del noroeste de México. En revisión.

32. FAO/W HO/UNU. Energy and protein requeriments. Report of a joint FAO/W HO/UN U Experts Consultation. Ginebra:W HO, (Technical Report Series núm. 74), 1985.

33. JamesW PT, Schofield EC. Human energy requirements. 0 xford:0 xford University Press, 1990

34.Voorrips LE,Van A cker TMCJ, D euerenberg P, van Staveren W A. Energy expenditure at rest and during standardized activities: A comparison between elderly and middle-aged women. Am J C lin N utr 1993;58:15-20. 35. Visser M, Van der Horst A, De Groot LCPGM, Deuerenberg P, Van Staveren W A. Energy cost of physical activities in healthy elderly women. Metabolism 1995;44:1046-1051.

36. Haggarty P,Valencia ME, Mcneill G, G onzález N L, Moya SY, Pinelli A et al. Energy expenditure during heavy work and its interaction with body weight. Br J N utr 1997;77:359-373.

37.Velázquez-Alva MC, C astillo-Martínez L, Irigoyen-C amacho E, ZepedaZepeda MA, G utiérrez-Robledo LM, C isneros-Moysen P. Estudio antropo- métrico en un grupo de hombres y mujeres de la tercera edad en la Ciudad de México. Salud Publica Mex 1996;38:466-473.

38. Mattila K, Haavisto M, Rajala S. Body mass index and mortality in the elderly. Bri Med J 1986;292:867-868.

39. Andres R, Elahi D, Tobin JD, Muller DC, Brant L. Impact of age on weight goals.Ann Intern Med 1985;103:1030-1033.

40. Committee on Diet and Health, Food and N utrition Board. Diet and health implications for reducing chronic disease risk. W ashington, D.C.: $N$ ational A cademy Press, 1989.

41. Reed RL, Yochum K, Pearlmutter L, Meredith KE, Mooradian AD. The interrelationship between physical exercise, muscle strength and body adiposity in a healthy elderly population. J Am Geriatr Soc 1991;39:11891193.

42. Teo G, Idris MN . Prevalence of hypertension among Chinese elderly and its relationship to behavioural and nutritional factors. Med J Malaysia 1996;51:33-40.

43. Masaki KH, C urb JD, C hiu D, Petrovitch H, Rodríguez BL.Association of body mass index with blood pressure in elderly Japanese American men. The Honolulu Heart Program. Hypertension 1997;29:673-677.

44. Manson JE, N athan D M, K rolewski AS, Stampfer MJ,W illetW C, Hennekens $\mathrm{CH}$. A prospective study of exercise and incidence of diabetes among US male physicians. JAMA 1992;268:63-67.

45. Manson JE, Rimm EB, Stampfer MJ, Colditz GA,W illett W C, Krolewski $A S$ et al. Physical activity and incidence of non-insulin-dependent diabetes mellitus in women. Lancet 1991;338:774-778.

46. King H, Finch C, Collins A, Koki G, King LF, Heywood P et al. G lucose tolerance in Papua $N$ ew Guinea: Ethnic differences, association with enviromental and behavioural factors and the possible emergence of glucose intolerance in a highland community. Med J Aust 1989;151:204-210.

47. Secretaría de Salud-Dirección General de Epidemiología. Encuesta N acional de Enfermedades Crónicas, 1993. México D.F.: SSA, 1993.

48. Castro V, Gómez-D antés H, N egrete-Sánchez J, Tapia-C onyer R. Las enfermedades crónicas en las personas de 60-69 años. Salud Publica Mex 1996;38:438-447.

49. Paffenbarger RS, Hyde TR, W ing AL, H sieh CC. Physical activity, allcause mortality and longevity of college alumni. N Engl J Med 1986;314:605613.

50. Rakowski W, Mor V.The association of physical activity with mortality among older adults in the Longitudinal Study of A ging (1984-1988). J Gerontol Med Sci 1992;47:M 122-129.

51. Paffenbarger RS Jr, Hyde TR,W ingAL, Lee IM, Jung D L, Kampert JB.The association of changes in physical-activity level and other lifestyle characteristics with mortality among men. N Engl J Med 1993;328:538-545. 